Review of Scientific Instruments, vol 74, 1, pp. 873-876 (2003)

\title{
Laser modulated optical reflectance of thin semiconductor films on glass
}

\author{
J.L.N. Fotsing, M. Hoffmeyer, S. Chotikaprakhan, D. Dietzel, J. Pelzl, B.K. Bein \\ (bein@ep3.ruhr-uni-bochum.de) \\ Exp.Phys. III, Solid State Spectroscopy, Ruhr University, D-44780 Bochum, Germany \\ F. Cerqueira, F. Macedo, J.A. Ferreira \\ Depart. de Física, Universidade do Minho, P-4709 Braga Codex, Portugal
}

\begin{abstract}
Semiconductor films, deposited by reactive magnetron sputtering on glass substrates have been analyzed with the help of laser-modulated optical reflectance. The results are discussed with respect to the thermal and charge carrier transport properties. Semiconductor properties have been identified both for micro-crystalline and amorphous films.
\end{abstract}

\section{Introduction}

Based on modulated Ar ion laser beam excitation and on the detection of the modulated optical reflectance by means of a He-Ne laser probe beam, a larger variety of semiconductor films, namely CdInSe-films, nano-crystalline and amorphous hydrogenated Si-based films deposited by RF sputtering on glass have been analyzed with respect to their thermal and electronic properties.

On semiconductor materials, the modulated reflectance signal in general depends on the superposition of the thermal wave and the charge carrier density wave [1]. Both can be separated in frequency dependent measurements due to the fact that the charge carrier diffusion, their recombination, and the thermal wave diffusion are governed by different time constants, and that for Silicon the temperature coefficient and the charge carrier density coefficient of the optical reflectance are opposite in sign. In layer systems consisting of thin semiconductor films on glass, however, the surface recombination can play the dominant role, contributing to surface heat sources only slightly retarded in time with respect prompt heating due to fast thermalization. This means the charge carrier diffusion effects on the measured signal may be relatively small and cannot be distinguished from the thermal wave effects.

Apart from the sensitivity with respect to semiconductor properties, another special advantage of the modulated optical reflectance technique is used here: it can be applied to film systems on glass, to measure and control the thermal and semiconductor properties of film deposition at the rear surface across the glass substrate.

In section 2 the samples and the conditions of film deposition are briefly described. In section 3 , results measured for a few examples of micro-crystalline and amorphous films are compared with reference signals of silicon and discussed with respect to the effective thermal and electronic transport properties.

\section{Description of samples and film deposition conditions}

The samples under investigation comprise thin semiconductor films, CdInSe films and nanocrystalline and amorphous hydrogenated Si-based films on glass substrates and compact wafer silicon as reference material. The analyzed Si-based films have been produced by reactive magnetron sputtering using an Alcatel SCM 650 chamber. The target material consisted of hyperpure silicon wafer material spaced at about $50 \mathrm{~mm}$ from the substrate support. For most 
of the samples, the sputter process has been run in a hydrogen-rich atmosphere, with variations of the RF power: $400 \mathrm{~W}, 200 \mathrm{~W}$, and $80 \mathrm{~W}$.

Previous work [2] has shown that low RF powers in a hydrogen-rich atmosphere contribute to thin films with silicon nanocrystals and that high RF powers contribute to completely amorphous material. X-ray analysis supplemented by Raman spectroscopy [3] confirmed that some of the films, e.g. sample b1, are amorphous and other films show silicon nano-crystals embedded in an amorphous matrix. The crystallinity has been characterized, e.g. for the film b2, by the crystal volume fraction of about $45 \%$ and an average crystal size between 50 - $60 \AA$ The film thickness has been determined from the transmission spectra by Swanepoel's method [4]: e.g. $1.05 \mu \mathrm{m}$ for $\mathrm{b} 2$, and $1.06 \mu \mathrm{m}$ for b1. The composition of the films has been obtained from ERD/RBS: about $56 \%$ of silicon, $25 \%$ of hydrogen, and $19 \%$ of oxygen.

\begin{tabular}{|c|c|c|c|c|c|c|c|c|c|c|}
\hline \multirow[t]{2}{*}{ Sample } & \multirow{2}{*}{$\begin{array}{c}\mathrm{RF} \\
\text { power/W }\end{array}$} & \multirow{2}{*}{$\begin{array}{l}\mathrm{T}_{\text {subst }} \\
{ }^{\circ} \mathrm{C}\end{array}$} & \multirow{2}{*}{$\frac{\mathrm{P}_{\mathrm{H} 2}}{\left(\mathrm{P}_{\mathrm{H} 2}+\mathrm{P}_{\mathrm{Ar}}\right)}$} & \multirow[t]{2}{*}{$\Delta \mathrm{t}$} & \multicolumn{2}{|c|}{ Cryst. size/Å } & \multirow{2}{*}{\begin{tabular}{|l|} 
Cryst. \\
vol \%
\end{tabular}} & \multirow[t]{2}{*}{$\mathrm{a} / \mathrm{c}$} & \multirow[t]{2}{*}{$\mathrm{d} / \mu \mathrm{m}$} & \multirow[t]{2}{*}{ Strain (\%) } \\
\hline & & & & & Raman & $\mathrm{X}$ ray & & & & \\
\hline Si 47 & 150 & 400 & 0.54 & 5000 & 72.5 & 96 & 65 & $\mathrm{c}$ & 1.17 & 3.5 \\
\hline Si 51 & 80 & 300 & 0.77 & & 60 & 115 & 50 & $\mathrm{c}$ & $<0.5$ & 2.2 \\
\hline S 52 & 150 & 150 & 0.77 & 54000 & & & & $\mathrm{a}$ & 3.88 & \\
\hline Si 53 & 80 & 300 & 0.77 & 30000 & 65 & 46 & 69 & $\mathrm{c}$ & $<0.5$ & 2.6 \\
\hline Si 54 & 81 & 400 & 0.57 & 22800 & 80 & 55 & 66 & $\mathrm{c}$ & 2.51 & 2.8 \\
\hline b1 & 400 & 200 & 0.17 & & & & & $\mathrm{a}$ & 1.06 & \\
\hline $\mathrm{b} 2$ & 200 & 250 & 0.17 & & 50 & 60 & 45 & $\mathrm{c}$ & 1.05 & \\
\hline
\end{tabular}

Table 1: Deposition conditions, crystallinity, film thickness, and crystal strain of samples

\section{Measurements of amorphous and microcrystalline semiconductor films on glass}

For the measurements, the standard equipment of photothermal microscopy based on the laser-modulated reflectance signal (Fig.1) has been used. Due to the fact, that the main interest in this work was not on imaging but on the frequency-dependence of the signals, relatively large radii $(15 \mu \mathrm{m})$ have been used both for the pump and the probe beam [5].

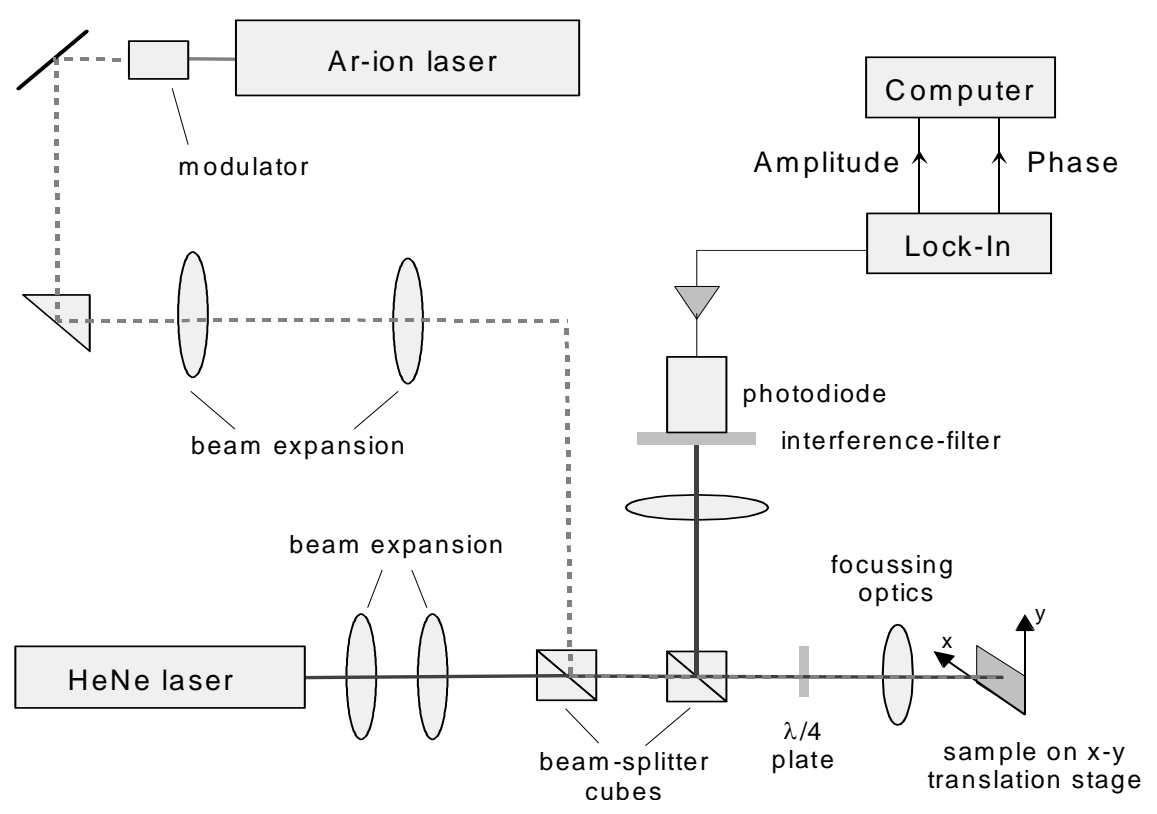

Fig. 1: Schematic of laser beam modulated optical reflectance

Subsequently some examples of modulated optical reflectance signals are shown, measured both at the front surface of the films and at their rear surface, as the interface film - glass substrate. 
In Figure $2 \mathrm{a}$ and $\mathrm{b}$ modulated optical reflectance signals of semiconductor films, measured across the glass substrate at the transition between substrate and film, are compared with the signals of a homogeneous Si sample (o). The signal amplitudes of the Si sample (Fig. 2b) are relatively small, showing the typical frequency characteristics of semiconductor materials: between $100 \mathrm{~Hz}$ and $20 \mathrm{kHz}$ the amplitudes (o) decrease with the growing frequency, above $20 \mathrm{kHz}$ they increase again. This is due to the fact, that the temperature coefficient $C_{\mathrm{T}}=$ $\partial R(T, n) / \partial T$ and the charge carrier coefficient $C_{\mathrm{n}}=\partial R(T, n) / \partial n$ for Si are opposite in sign, and that the thermal wave contributions at low frequencies are slightly larger than the plasma wave contributions. While the thermal wave contributions decay in the limit of high modulation frequencies according to $f^{-1 / 2}$ the charge carrier contributions become dominant, leading to the increase of the amplitudes for $f>20 \mathrm{kHz}$. The amplitudes (Fig. 2b) of the amorphous Si-based layer (b1 $*$ ) also show a behavior typical for charge carrier contributions: In the range between about $1-10 \mathrm{kHz}$, they strongly decay with the frequency, and above $f>$ $10 \mathrm{kHz}$ the decrease is less pronounced, indicating comparatively larger charge carrier contributions at higher frequencies.
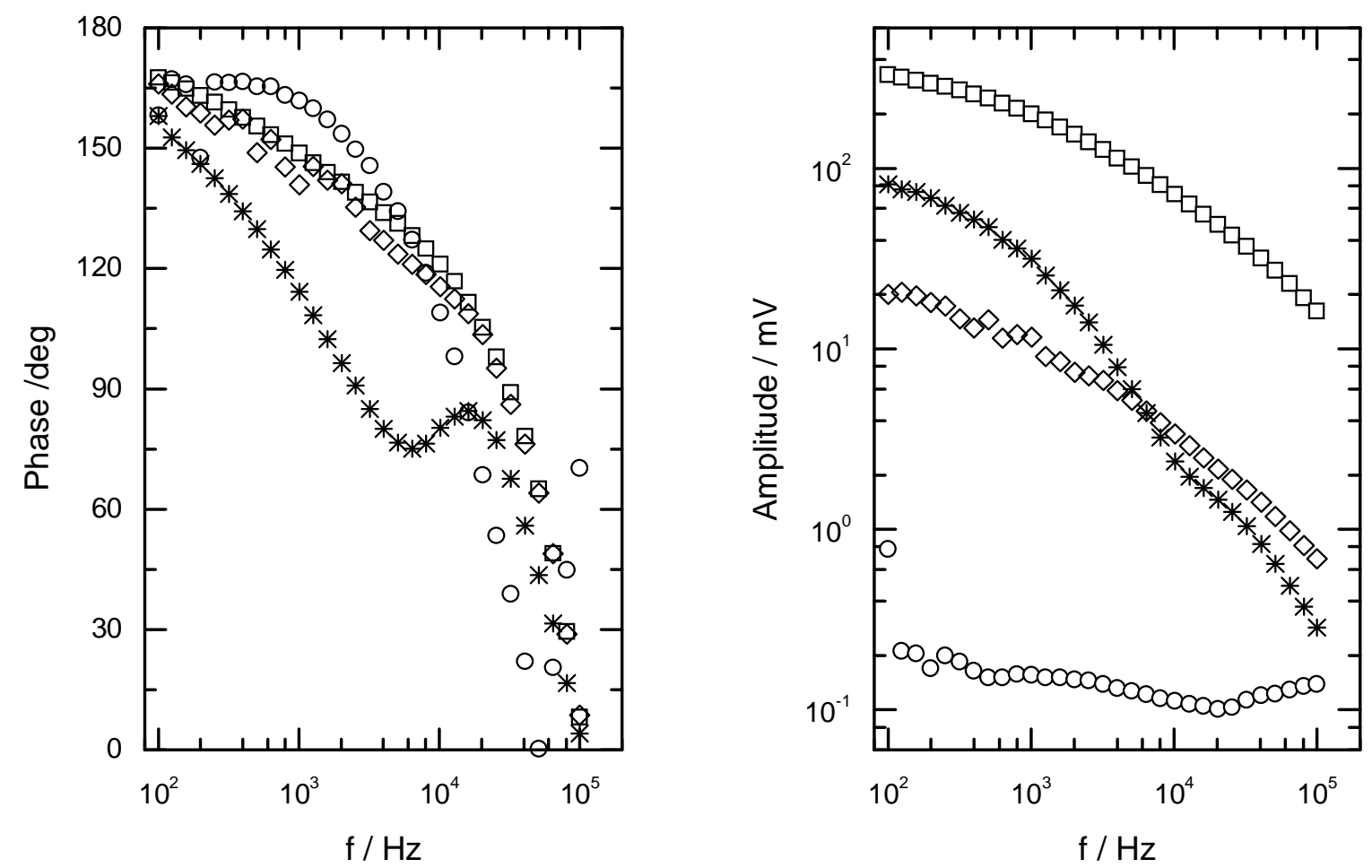

Figure 2a and b: Modulated optical reflectance signals, phases (a) and amplitudes (b), measured at the transition between semiconductor film and glass substrate for Si-based films (b2 $\square$, b1 *) and a CIS film $(\diamond)$ on glass, in comparison with signals measured for compact Silicon $(\mathbf{0})$.

While the amplitudes of the micro-crystalline Si-based film b2 ( $\square)$ and of the CIS film $(\diamond)$ in Fig. $2 \mathrm{~b}$ do not give any special hint with respect to possible charge carrier contributions, such contributions become evident in the phases (Fig. 2a): At low and intermediate frequencies, the phases of the two samples $(\square, \diamond)$ first decrease by about $60^{\circ}$, from $165^{\circ}$ at $100 \mathrm{~Hz}$ to a value of about $105^{\circ}$ at $20 \mathrm{kHz}$. This is characteristic for thermal wave contributions in semitransparent systems. Above $20 \mathrm{kHz}$ a further decay of the phases of about $105^{\circ}$ can be observed. This behavior is characteristic for small charge carrier contributions to the modulated reflectance signal, which only above $20 \mathrm{kHz}$ become comparable with the thermal 
wave contributions or become even dominant. The negative sign of the charge carrier coefficient $C_{\mathrm{n}}$ then leads to a phase retardation of $-180^{\circ}$, a value not achievable by thermal wave contributions.

The phases of the amorphous film b1 (*) in Fig. 2a deviate significantly from the behavior of the other samples: At low frequencies they already show a strong decay, between about $6 \mathrm{kHz}$ and $15 \mathrm{kHz}$, however, they increase again, and finally at higher values they continuously decrease again. From this behavior we conclude that two significant signal contributions may contribute at low frequencies, namely heat sources due to charge carrier recombination at the transition between Si-based film and glass, where the modulated reflectance signal is measured, and a second retarded contribution due to surface recombination at the opposite surface, the front surface of the film. Owing to the low thermal diffusivity and the thickness of the film, the effect of charge carrier recombination at the front surface on the signal measured at the rear surface is increasingly damped above about $6 \mathrm{kHz}$, and for $f>20 \mathrm{kHz}$ only the recombination effects at the transition between Si-based film and glass substrate can contribute to the measured signal.

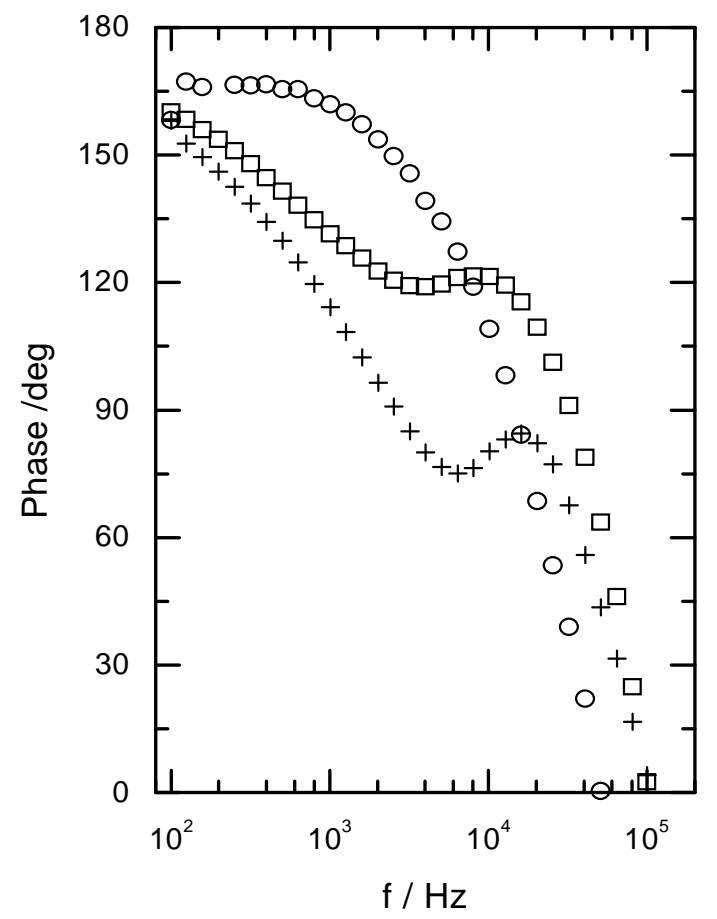

Figure 3: Phases of the modulated optical reflectance signals, measured for the amorphous

Si-based film b1 at the front surface $(\square)$ and at the transition $(+)$ between film and glass substrate, compared with phases for compact $\mathrm{Si}(\mathbf{0})$.

In Figure 3 the phases measured at the front surface ( $\square$ ) of the amorphous film b1 and those measured at the transition between Si-based film and glass substrate (+) are compared. In principle, the phases behave similarly, however, with a shift of the relative minimum, $\varphi_{\min } \leq$ $120^{\circ}$, and maximum $\varphi_{\min } \geq 120^{\circ}$ to lower frequency values, $f_{\min } \approx 3 \mathrm{kHz}$ and $f_{\max } \approx 9 \mathrm{kHz}$, which may be due to differences of the charge carrier recombination process at the two surfaces and asymmetries in the thermal attenuation. 
Comparing the phases measured for the amorphous film b1 at the front surface ( $\square$ ) and at the transition to the glass substrate $(+)$ in Fig. 3 with the corresponding values measured for sample Si $47 \mathrm{bm}$ (Fig. 4a) at the front surface (0) and at the transition to the glass substrate at $(-)$, we can see that the phases of sample $\mathrm{Si} 47 \mathrm{bm}$ at the front surface show a much stronger articulation, with a relative minimum $\varphi_{\min } \approx 110^{\circ}$ at $f_{\min } \approx 200 \mathrm{~Hz}$ and a (second) relative maximum $\varphi_{\max } \approx 135^{\circ}$ at $f_{\min } \approx 4 \mathrm{kHz}$.

The phases measured at the interface between film and glass substrate of sample $\mathrm{Si} 47 \mathrm{bm}$ show a comparatively much smaller articulation, only with a turning point at about $6 \mathrm{kHz}$. The phase shift over the whole measured frequency $(10 \mathrm{~Hz}-100 \mathrm{kHz})$, however, is much larger (about $-240^{\circ}$ ) than that found at the front surface (about $-140^{\circ}$ ).
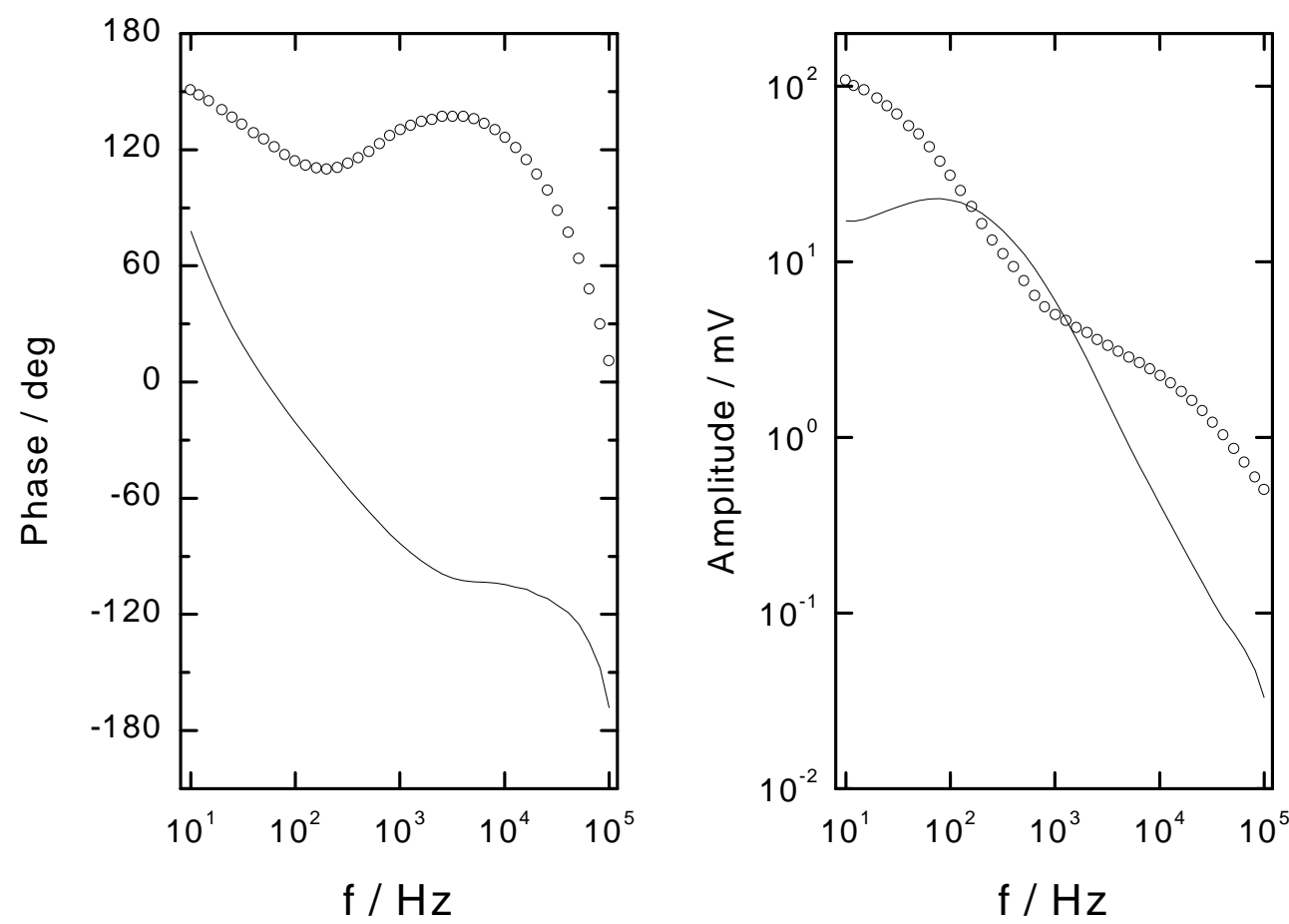

Figure 4a and b: Modulated optical reflectance signals, phases (a) and amplitudes (b), measured as function of frequency at the front surface (o) and at the transition to the glass substrate at (-) of a thin Si-based film Si $47 \mathrm{bm}$.

Comparing the amplitudes measured for sample $\mathrm{Si} 47 \mathrm{bm}$ at the front surface (0) and at the rear surface, at the transition to the glass substrate (--), we can see that the amplitudes of the modulated optical reflectance signal at the rear surface, in contrast to the amplitudes measured at the front surface, first increase with the frequency and reach a relative maximum at about $100 \mathrm{~Hz}$. This may be explained by a plasma wave contribution at the rear surface which is larger than the thermal wave contribution. Such a dominance of the plasma wave contribution is confirmed by the overall phase shift of $-240^{\circ}$ observed for the rear surface phases in Fig. 4a.

Phases and amplitudes with considerable variations of the slope as function of frequency have also been found on sample Si $53 \mathrm{bc}$ (Fig. 5a and b). In contrast to sample Si $47 \mathrm{bm}$, the amplitudes at the transition between Si-based layer and glass substrate, at the rear surface of the film (-), are larger than those measured at the front surface $(\Delta)$ and show a quite regular decrease, without any spectacular changes of the slope (Fig. 5b). The amplitudes measured at the front surface $(\Delta)$ show a turning point at about $9 \mathrm{kHz}$ (Fig. 5b) and the corresponding 
phases show a relative minimum $\left(\varphi_{\min } \leq-120^{\circ}\right)$ at about $9 \mathrm{kHz}$ and a relative maximum $\left(\varphi_{\max } \geq-120^{\circ}\right)$ at about $30 \mathrm{kHz}$ (Fig. $\left.5 \mathrm{a}\right)$.

If indeed the relative phase minima are related to charge carrier recombination heat sources at the opposite surfaces of the films, the frequency values $f_{\min }$ of the relative phase minima measured at the front surfaces of the sample Si 53 bc (Fig. 5a) and Si 47 bc (Fig. 4a) give information on the thermal diffusion time of the films. Once the film thickness is known (Table 1 ), the ratio of the thermal diffusivities can be estimated, e.g. $\alpha_{\mathrm{Si53}} \approx 7.3 \cdot \alpha_{\mathrm{Si} 47}$.
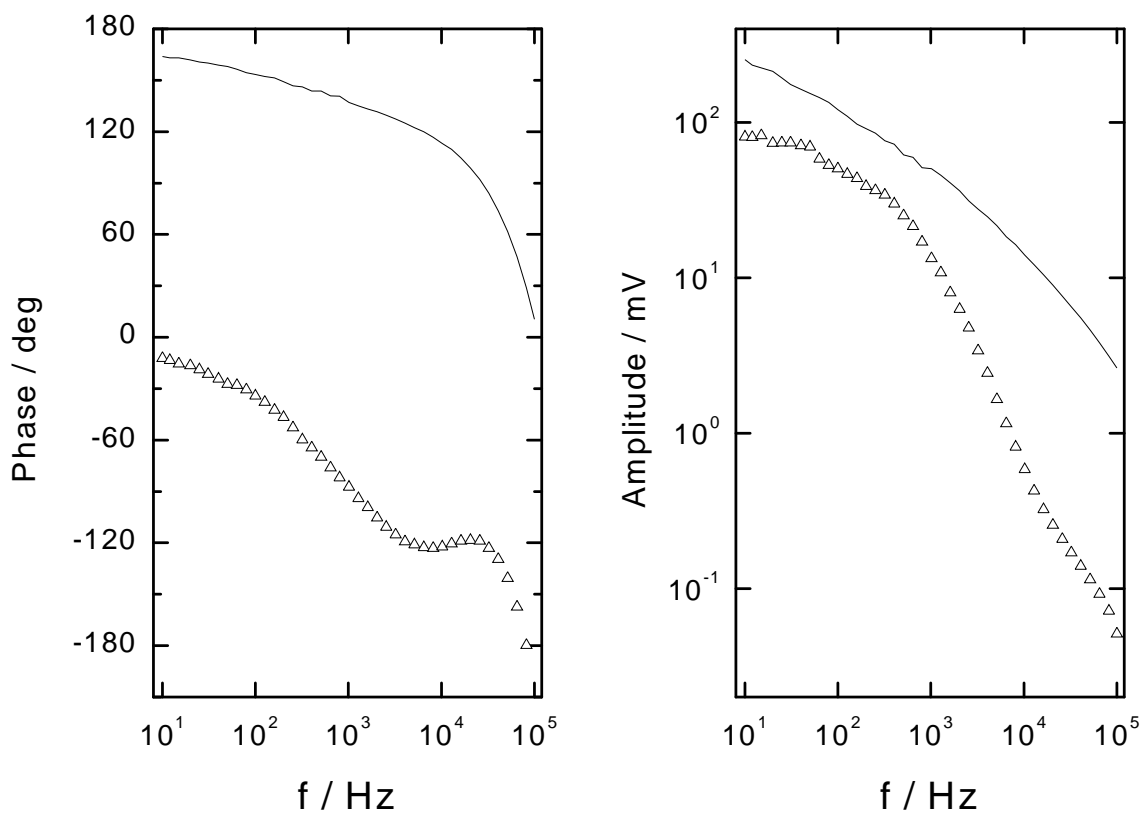

Fig. 5a and b: Modulated optical reflectance signals, phases (a) and amplitudes (b), measured as function of frequency at the front surface $(\Delta)$ and at the transition to the glass substrate (--) of a thin Si-based film Si 53 bc.

\section{Conclusions and Outlook}

Owing to the reduced film thickness $(0.5-3 \mu \mathrm{m})$ and the large number of physical parameters involved in the problem of coupled charge carrier diffusion and thermal wave diffusion, it is difficult to identify the effects of the different parameters on the measured signals. To overcome this problem, the reflectance signals and modulated IR transmission signals [6], measured both at the front and the rear surface of the samples, have to be combined. By this procedure the number of unknown parameters can be reduced, and the range of the numerical values of the parameters can be limited.

\section{References:}

[1] D. Fournier, in: D. Bicanic (Ed.), Photoacoustic and Photothermal Phenomena III, Springer Ser. Opt.Sci. 69 (1992), 339.

[2] M.F. Cerqueira, J.A. Ferreira, J. Materials Processing Technology 92-93 (1999), 235-238.

[3] M.F. Cerqueira, M. Andritschky, L. Rebouta, J.A. Ferreira, M.F. da Silva, Vacuum 46 (1995), 1385-1390.

[4] R. Swanepoel, J. Phys. E 16 (1983), 1214.

[5] D. Dietzel, J. Pelzl, B. K. Bein, Systematic analysis of laser beam modulated optical reflectance signals, this conference, to be published in 
[6] D. Dietzel, J. Gibkes, S. Chotikaprakhan, B.K. Bein, J. Pelzl, Radiometric analysis of laser modulated IR properties of semiconductors, to be publ. J. Thermophysics, 2002. 